\title{
Predicting the causes of operational anomalies in blade coating
}

\author{
P. Alam, C. Stoor \& M. Toivakka \\ Abo Akademi University, Turku, Finland
}

\begin{abstract}
Blade coating is a high speed $\left(\sim 20 \mathrm{~ms}^{-1}\right)$ coating operation that involves the squeezing of shear thinning coating slurry between a base paper substrate and a steel metering blade. The gap between the blade and the paper can be as small as $1 \mu \mathrm{m}$ and mineral pigments in the slurry can become trapped, giving rise to streaks and scratches on the coating surface. The objective of the research presented herein is to develop a model for simulating the process of blade metering and to use the model to investigate operational anomalies connected to the process. The effects of specific variables were studied notably; blade shape and backing roll stiffness. Virtual experimentation was undertaken to study the deformation of the paper surface, the coating colour thickness and the position of the stagnation point (where pigments may gather). The choice of blade type was found to have a highly localised influence on the area close to the nip. The backing roll stiffness was shown to be one of the most important parameters in blade metering. Lowering the stiffness decreases the coating thickness and pushes the stagnation point further away from the heel region.
\end{abstract}

Keywords: solid mechanics, fluid dynamics, multiphysics, modelling simulation, metering, coating, time dependent non-Newtonian hyperelastic process engineering.

\section{Introduction}

The coating of paper is a process by which coating colour is applied to one or both sides of the paper. Paper and board are primarily coated in order to improve the optical and printing properties. Though, functional coatings might be coated on a variety of speciality papers, such as greaseproof paper for food packaging [1]. Blade coating is currently the most commonly used method for pigment 
coating of paper and board. Its popularity can be explained by some of the advantages blade coating offers. High web speeds, good surface smoothness of the coating layer and high dry solids content used in the coating colour are all benefits that make blade metering a most attractive option. Metered size press is, however, gaining ground in the lightweight coated paper market and there is moreover growing interest in curtain coating [2].

The principle set up of blade coating with an applicator roll is illustrated in Figure 1. The diameters of the rolls are determined by the speed at which the operation is conducted. Higher web speeds and wider machines require larger diameters. The backing roll diameter for big and fast machines can therefore come up to $1500 \mathrm{~mm}$, whereas for small and slow machines, the diameter is around $600 \mathrm{~mm}$. The applicator roll diameter is typically $30-40 \%$ that of the backing roll. It is located directly under the backing roll, or is slightly offset from it. The applicator roll rotates at a speed between $15-25 \%$ that of the machine speed, with a gap of $0.5-1.5 \mathrm{~mm}$ between the rolls. The rolls are normally covered with rubber, a polymer based composite or sometimes with polyurethane.

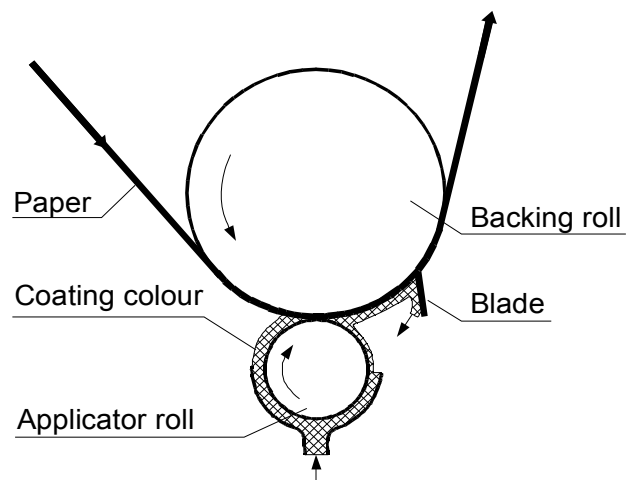

Figure 1: $\quad$ Principle set up of blade coating with an applicator roll.

The coating colour is an aqueous suspension consisting of several components. The pigment is volumetrically preponderant reaching $80-95$ wt. $\%$ of the dry coating. Minerals are usually used as pigments, commonly kaolin clay and calcium carbonate, but synthetic pigments are also available. Nearly all coating pigment particles have a diameter that is less than $10 \mu \mathrm{m}$ [3].

In the applicator nip and under the blade the colour experiences a pressure pulse, causing a filtration process on the paper surface to take place. The liquid phase of the colour penetrates into the paper at an accelerated rate, consequently forming a thickened layer of consolidated pigment. The layer is termed the filter cake and grows continuously from the application of colour. In the absence of the pressure pulses, during the dwell time, it is capillary absorption that causes the growth of the filter cake. After metering, the coating colour on the paper comprises a dewatered filter cake of several micrometers thickness and a liquid layer of colour on the cake surface. 
Bleeding is a build-up of coating colour on the side of the blade ordinarily free from contact with the colour. Bleeding results in the formation of stalagmites, whiskers or beards. The type of deposits formed depends upon the heights reached by the coating colour on the blade. The deposits can become detached, impairing the quality of the surface or even causing web-breaks. The principle cause of bleeding is suggested to be related to excessive dewatering of the coating colour between application and metering [4]. All circumstances that lead to a colour of high solids content and viscosity at the edge of the blade may be a cause for bleeding. This includes colour with poor water holding properties and colour with shear thickening problems. Additionally, overly high blade pressures can force considerably high amounts of fluid into the base paper [5].

Other operational parameters believed to induce bleeding are high web speeds and unfavourable metering geometries. A uniformly converging metering nip with a sharp edge is considered the ideal metering geometry. Diverging metering nips cause bleeding even at low coater speeds. Flow disturbances under the blade may lead to colour drops being ejected at the blade tip, providing a nucleating site for bleeding or stalagmite formations. The deformation of the paper and backing roll in connection with the tip angle and bending of the blade is thus of significant importance [4].

Streaks are lines, scratches or indentations in the coating surface. They vary in width from less than $3 \mathrm{~mm}$ up to $6 \mathrm{~mm}$ and above. The streaks typically stretch tens of meters in the machine direction. Indentations can be caused by foreign material caught under the blade. These could include dried coating lumps, fibres, paper from breaks and poorly dispersed particles. Another reason for streaks may be particles adhering to the blade resulting in a localised build-up of immobile colour. This in turn could be connected to a low blade pressure or an improper blade angle. These indentations are called blade scratch, blade streaks or coating streaks. Another type of streaking is wet streaks, defined as heavy lines of coating in the machine direction. They appear glossier, more opaque and are less receptive to ink than the general coating. Wet sheet or localised low blade pressures are some of the causes [5].

\section{Procedure}

Comsol Multiphysics 3.2 was used in the preprocessing, solving and postprocessing stages of this research.

\subsection{Blade geometries}

Figure 2 shows schematics for two double bevelled blades and a single bevelled blade. The blade lengths are the same in each, as are the angles of the first bevel. The default toe angle is $2^{\circ}$ and the blade thicknesses are $540 \mu \mathrm{m}$.

The coating colour, filter cake, base paper and backing roll are also built into the model. Figure 3 indicates the dimensions, which were kept constant throughout the different simulations.

Due to the high pressure pulse under the blade, the filter cake is believed to grow more rapidly there. The filter cake grows within a distance of $2 \mathrm{~mm}$ in the 


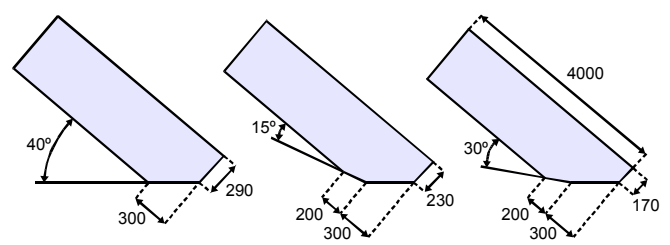

Figure 2: $\quad$ Blade geometries $(\mu \mathrm{m})$ for double and single bevelled blades.

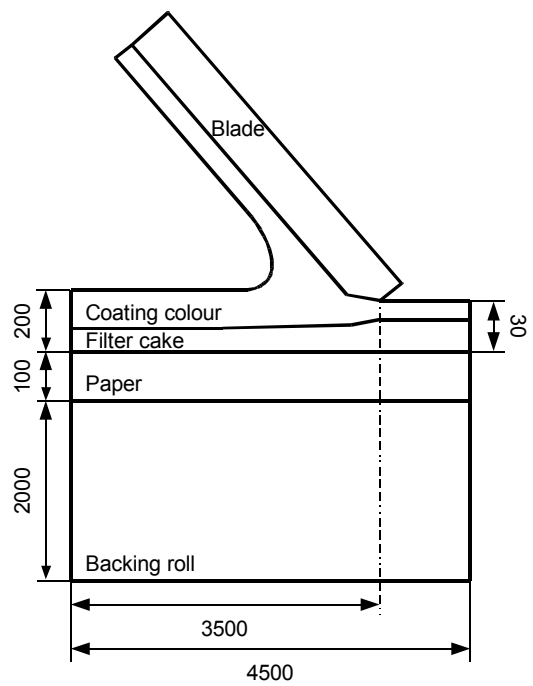

Figure 3: Dimensional constants $(\mu \mathrm{m})$ in all models for all materials.

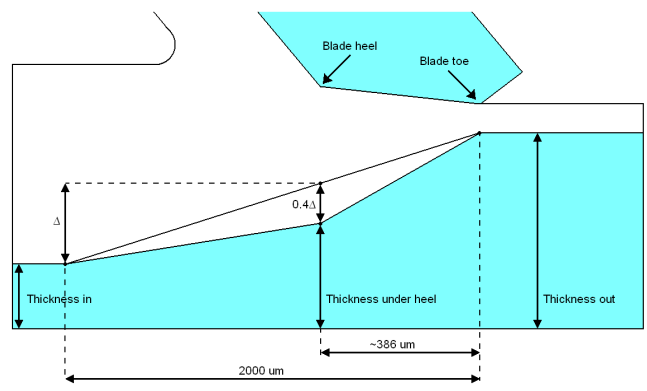

Figure 4: $\quad$ Modelled filter cake growth for single bevelled blades.

primary direction of coating flow, ending at the blade toe. This increased growth ratio used in the models is illustrated in Figure 4 for the single bevelled blade and in Figure 5 for the double bevelled blades. 


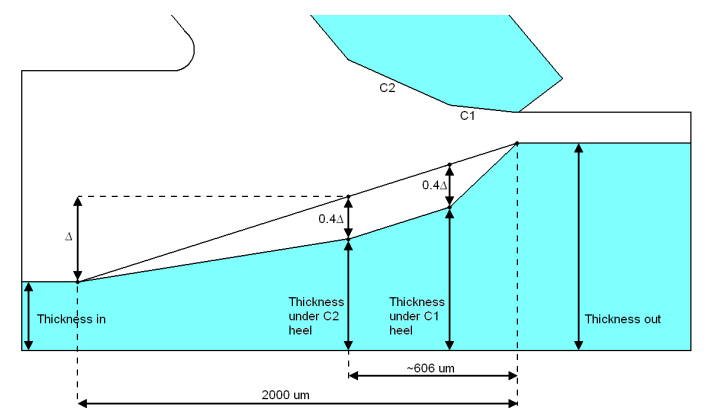

Figure 5: $\quad$ Modelled filter cake growth for double bevelled blades.

\subsection{Application modes}

Both the Power law model and the Carreau model were used in this work. Table 1 gives the properties assigned for the Power Law colour. The density of the colour, for both of the models, was chosen to be the one used in [6]. These models describe shear thinning coating colours. The application is based on the incompressible Navier-Stokes equations. The dependent variables are the velocity in the $\mathrm{x}$ direction, the velocity in the $\mathrm{y}$ direction, pressure and Lagrange multipliers that describe boundary fluxes, which in the case of the NonNewtonian Flow application is the total force. Table 1 gives the properties assigned for the Power Law colour. The density of the colour, for both of the models, was chosen to be the one used in [6]. The power law model describes a shear thinning coating colour.

Table 1: $\quad$ Power law parameters, from [7].

\begin{tabular}{|c|c|}
\hline Power law & Coating colour \\
\hline$\rho\left[\mathrm{kg} / \mathrm{m}^{3}\right]$ & 1400 \\
\hline$m[\mathrm{Pas}]$ & 1 \\
\hline$N$ & 0.9 \\
\hline
\end{tabular}

Table 2 gives the parameters used by [8] in their Carreau-fit to measured experimental data. The first is a colour with a high Brookfield viscosity of 700 $\mathrm{mPas}(100 \mathrm{rpm})$. The second colour has a low Brookfield viscosity of $100 \mathrm{mPas}$ (100 rpm).

All solids were modelled as 2-dimensional plane strain materials. The dependent variables include displacement in the $\mathrm{x}$-direction, displacement in the $\mathrm{y}$-direction, and pressure. The backing roll was taken to be hyperelastic and modelled as a Mooney-Rivlin material. Table 3 gives the constants C10 and C01 taken from the study of [9]. At small strains the hyper-elastic material is 
comparable to a Hookean elastic material with an elastic modulus of around $200 \mathrm{MPa}$. The value is in accordance with the elastic modulus of $150 \mathrm{MPa}$ used by [10]. The density was set to $1100 \mathrm{~kg} / \mathrm{m}^{3}$.

Table 2: $\quad$ Carreau parameters, from [8].

\begin{tabular}{|c|c|c|}
\hline Carreau & High viscosity colour & Low viscosity colour \\
\hline$\rho\left[\mathrm{kg} / \mathrm{m}^{3}\right]$ & 1400 & 1400 \\
\hline$\eta_{\infty}$ & 0.038 & 0.001 \\
\hline$H_{0}$ & 0.728 & 0.104 \\
\hline$\Lambda$ & 0.046 & 6.878 \\
\hline$s$ & 0.695 & 0.944 \\
\hline
\end{tabular}

Table 3: $\quad$ Backing roll parameters, from [9].

\begin{tabular}{|c|c|}
\hline Mooney Rivlin & Backing roll \\
\hline$\rho\left[\mathrm{kg} / \mathrm{m}^{3}\right]$ & 1100 \\
\hline$C_{10}[\mathrm{~Pa}]$ & $14.14 \times 10^{6}$ \\
\hline$C_{01}[\mathrm{~Pa}]$ & $21.26 \times 10^{6}$ \\
\hline
\end{tabular}

All other solids in the model were taken to be isotropic Hookean elastic materials and are summarised in Table 4. Mann et al. [11] conducted a thorough research on the mechanical properties of machine-made paper. Based on their work, the properties of the base paper were chosen. The filter cake density was set to $2000 \mathrm{~kg} / \mathrm{m}^{3}$, with an elastic modulus of $200 \mathrm{MPa}$ and a Poisson's ratio of 0.30 . The blade was given the values of structural steel (210GPa elastic modulus and 0.33 Poisson's ratio).

Table 4: $\quad$ Isotropic material properties.

\begin{tabular}{|c|c|c|c|}
\hline $\begin{array}{c}\text { Isotropic } \\
\text { material }\end{array}$ & $\begin{array}{c}\text { Base paper, from } \\
{[11]}\end{array}$ & Filter cake & Blade \\
\hline$\rho\left[\mathrm{kg} / \mathrm{m}^{3}\right]$ & 1000 & 2000 & 7850 \\
\hline$E[\mathrm{~Pa}]$ & $3.90 \times 10^{7}$ & $2.00 \times 10^{8}$ & $2.00 \times 10^{11}$ \\
\hline$v$ & 0.0018 & 0.30 & 0.33 \\
\hline
\end{tabular}

An Advanced Lagrange Eulerian (ALE) method was implemented to allow greater flexibility in mesh movement. The dependent variables were the mesh positions, $\mathrm{x}$ and $\mathrm{y}$, and the Lagrange multipliers. The independent variables of the whole system were the space variables X and Y. Mesh movement followed 
the movement of the physical material. The coating colour is allowed free displacement and all solid materials are deformed due to physics induced displacements.

\subsection{Boundary conditions}

The driving force of the whole simulation is the moving filter-cake surface. The $1400 \mathrm{~m} / \mathrm{min}$ web velocity acts as the driver for coating colour flow. Both the top of the blade and the bottom of the backing roll are constrained (zero degrees of freedom). These boundaries are not allowed to move or deform. The sides of the backing roll, base paper and filter cake are allowed to deform only in the ydirection. These restrictions of movement put on the solids keep the system "in place" and reduces the number of degrees of freedom (DOF) for the whole model.

The colour is in contact with the blade and the filter cake. These boundaries between solid and fluid are of great importance. The solids are subjected to forces by the fluid, edge loads, which deform the solids. In turn, the moving mesh and the fluid move with the solids and deform along with it. The coupling is managed using the Lagrange multiplier of the fluid together with the displacement variable of the solid. Slip conditions under the blade can be induced by predefining a slip velocity on the lower surface of the blade. An alternative approach could be to define slip velocity as a function of local tangential shear stress. After the blade toe, the moving mesh is free to move on the surface of the applied coating layer. This acts essentially as a free surface without the inclusion of surface energetics.

\section{Results}

Figure 6(a) shows the maximum vertical deformation plotted for each of the blade types. The deformation can be seen to increase as the $\mathrm{C} 2$ angle is increased with double bevelled blades causing greater deformation than single bevelled blades. Figure 6(b) presents a clear shift of the maximum point of deformation away from the toe as the double bevel C2 angle is increased. Moreover both double bevelled blades have an $\mathrm{x}$ Cartesian maximum point of deformation farther from the blade toe than the single bevelled blade. In this model, this phenomenon is implicitly related to the modelled filter cake growth. As the C2 bevel angle is increased, the gradient of the filter cake under the $\mathrm{C} 1$ bevel heightens. The single bevel has the lowest cake gradient. Figure 7 describes the mechanics of fluid movement and its effect on the surrounding materials. As the gradient of the filter cake increases, there is a shift in force scalars and the resulting hydrodynamic lifting forces increase. This causes greater material deformation. Having the maximum compression further away from the critical toe region should be considered to be more beneficial. The region of maximum displacement will change the flow profile of the fluid the most. If this region is close to the tip of the blade a flow directed upwards may eject colour up on the blade tip. 

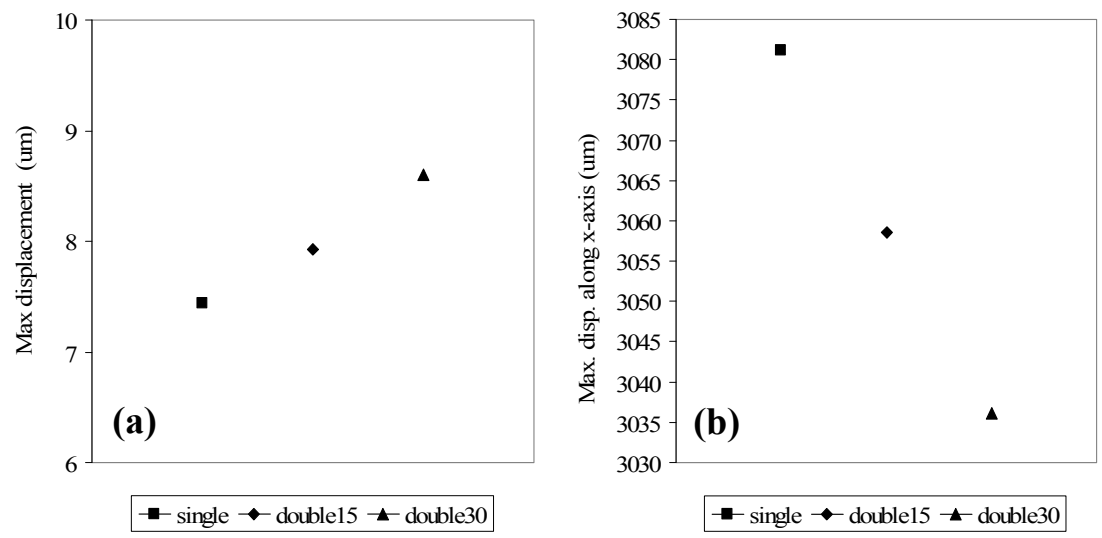

Figure 6: (a) Maximum deformation plotted for each of the blade types and (b) maximum displacement along the $\mathrm{x}$-axis plotted for each of the blade types.

Relatively small changes in the backing roll stiffness produce the largest deformations after the blade toe, approaching $4 \mu \mathrm{m}$ for the softest backing roll. Alam and Toivakka [12] highlighted the possibility that paper buckling at the outlet might alter flow paths and give rise to operational anomalies such as bleeding. Coating colour folding over the blade toe is therefore to be considered most likely in the case of a low stiffness backing roll. Figure 8(a) shows the increase in the maximum displacement as a function of lower $\mathrm{C} 10$ and $\mathrm{C} 01$ values. Figure 8(b) suggests there is a very subtle shift of the maximum displacement point from the tip of the blade as the $\mathrm{C} 10$ and $\mathrm{C} 01$ values are reduced.

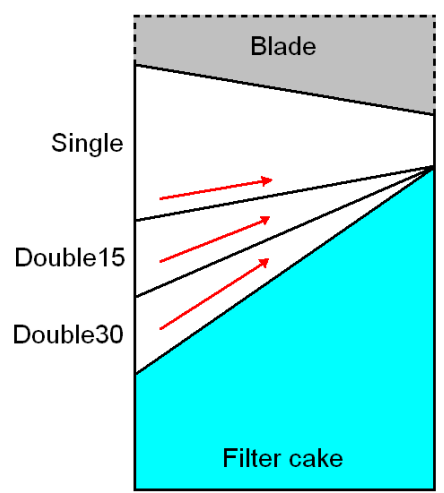

Steeper filter cake gradient causes alteration in the force scalars (orange arrows) resulting in heightened hydrodynamic forces and consequently greater deformation.

Figure 7: Illustrating the mechanics of fluid-structure interactions for single and double bevelled blades. 
Figure 9(a) shows that for a lower stiffness material, there exists a decrease in the coating thickness. An increase in the distance between the stagnation point and the heel of the blade is furthermore seen to arise on lowering the backing roll stiffness, Figure 9(b). Bousfield and co-workers [13] showed that lower stiffness rubber rolls will increase the necessary force on the blade needed to obtain the same target coat weight. The study was a comparison between a non-deformable backing roll and a backing roll with a Young's modulus of $5 \mathrm{MPa}$. In this work a much narrower region is investigated and the results show an opposing trend to that of [13].
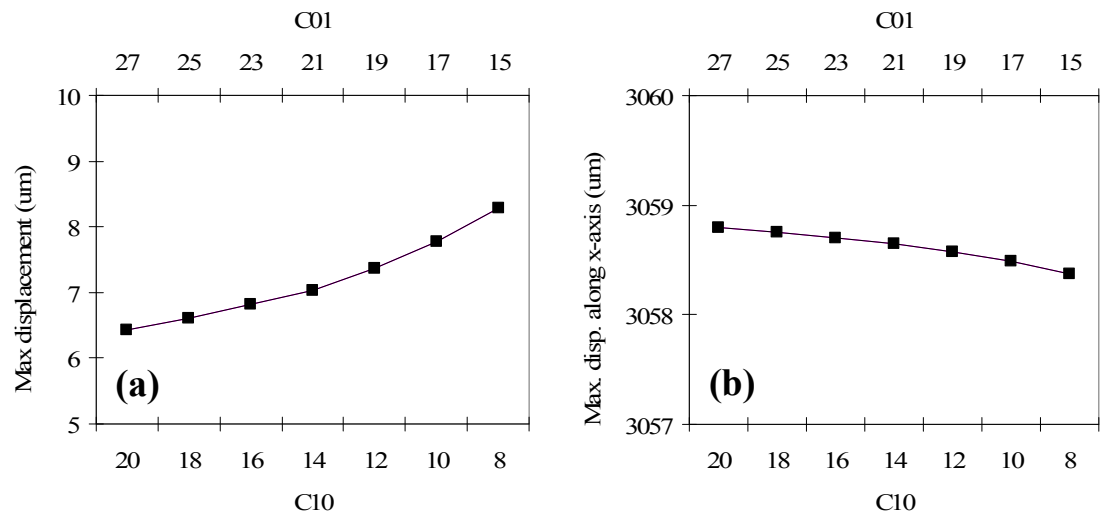

Figure 8: (a) Maximum displacement and (b) maximum displacement along $\mathrm{x}$-axis plotted as a function of decreasing backing roll stiffness.
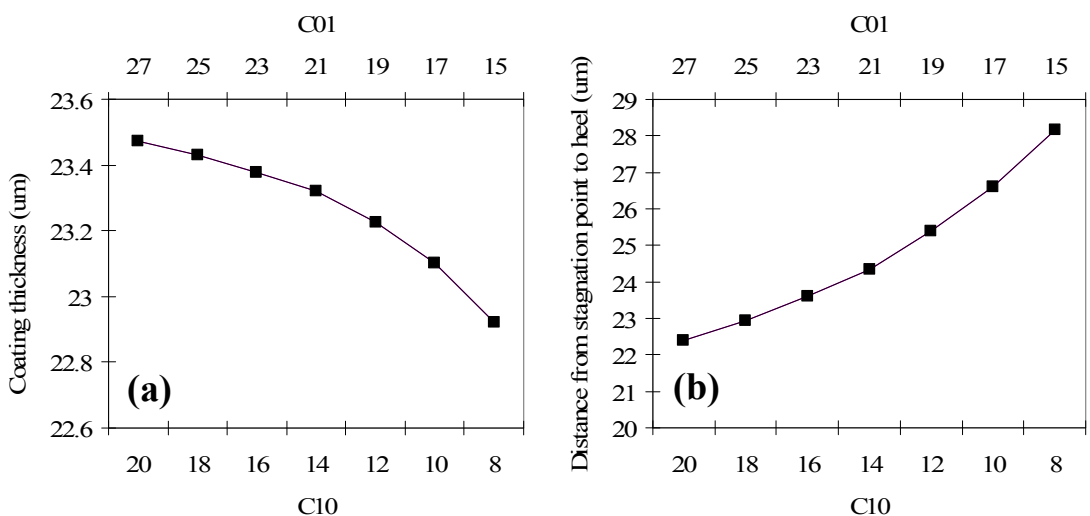

Figure 9: (a) Coating thickness and (b) distance from stagnation point to the heel plotted as a function of decreasing backing roll stiffness. 
Figure 10 shows that the total force acting on the blade decreases non-linearly as a function of a lowered backing roll stiffness. The forces are calculated by integrating the total force per unit area over the blade-colour boundaries. The force vector has a direction of approximately 54 degrees to the paper surface, i.e. in the direction of the deflection of the blade, with only a small variation due to the stiffness of the backing roll.

The disagreement in results with [13] are believed to be connected to an understanding of the hydrodynamic lifting force. Hydrodynamic bearing is a function of the fluid viscosity, the machine speed, the wedge angle and a coefficient including the geometry of the wedge. When only the backing roll stiffness is varied it is the shape of the wedge-like channel forming between the blade and the substrate that determines the magnitude of the lifting force. The lifting force is present for a converging wedge, not for a parallel channel between blade and substrate.

However, the hydrodynamic force as a function of the wedge angle has a maximum value at an angle of $3^{\circ}$ [1]. In Figures 11(a) and 11(b) the initial positions of the solids are shown by the contours. The deformed solids are filled with colour. The deformation is scaled by ten in order to be more illustrative, the wedge angle is as a result exaggerated. The softer backing roll has greater wedge convergence, which will reduce the hydrodynamic lift when a maximum point has been surpassed. Notably, the deflections of the two blades are approximately equal. The backing roll however, is raised somewhat for the softer rubber. Compression of the base paper is also noticeable in the figures. Both the forces acting on the blade and the coating thickness reduces therefore with a softer backing roll.

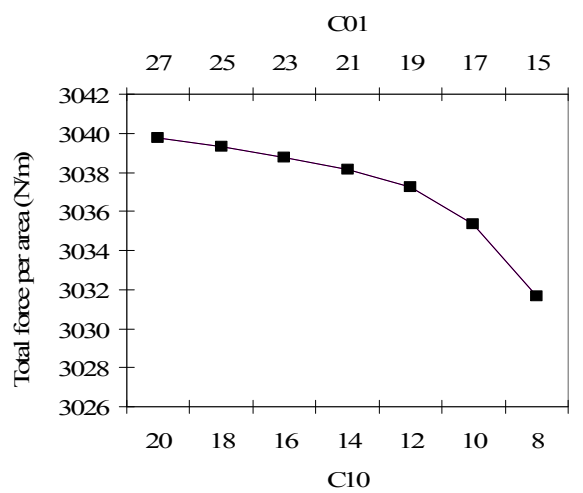

Figure 10: Total force per unit area plotted against backing roll stiffness.

An interesting phenomenon is the simultaneous increase in the stagnation point with a decrease in the coating thickness. It may be reasonable to think that a stagnation point located higher up and further away from the heel is evidence for an increased volume of flow under the blade. However, the stagnation point is relative to the blade and will not take into account properties such as gap size 
or toe angle. The stagnation point describes a highly localised area of the coating colour and conclusions are to be drawn carefully. In Figure 12 two scenarios are illustrated. Two particles start from the same position and end up at two different stagnation points. The particles will follow different paths due to, for instance, different pressures in the fluid. Initially however, the same amount of fluid exists under the streamlines and therefore the same coat weight is applied. A higher stagnation point is nevertheless preferable since streaking and scratching defects are thought to be linked to the region around the stagnation point [14]. Iliopoulos and Scriven [15] discussed the possibility of particles entrapment in shear thinning suspensions where there are low shear rates and relatively high viscosities. For a higher stagnation point particles flowing near the blade are subjected to prolonged shear rates, which in turn reduces the risk of particle trapping and aggregation. Optimising the backing roll stiffness is therefore a viable means of controlling the position of the stagnation point.
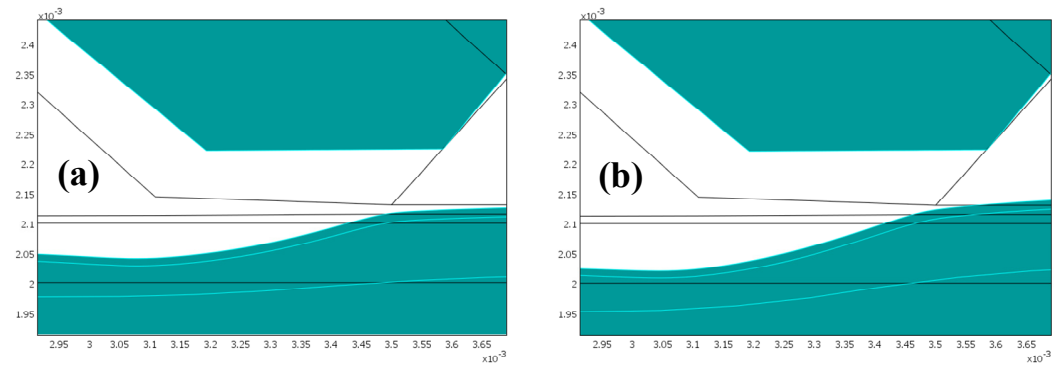

Figure 11: Deformed solids (scaled by 10) and the shape of the wedge that develops for (a) harder backing roll material and (b) softer backing roll.

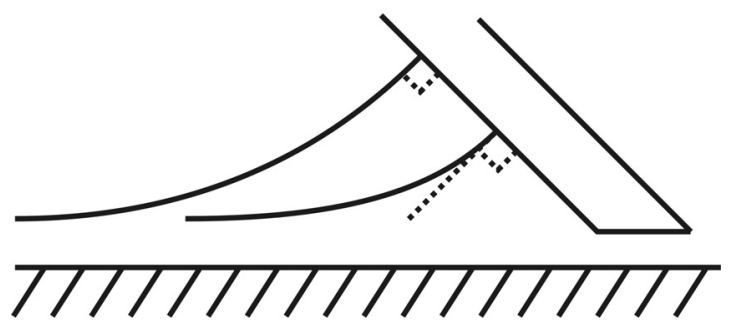

Figure 12: $\quad$ Sketch illustrating different stagnation point positions.

\section{Conclusions}

A multiphysics model has been developed to better understand how operational anomalies may arise in metering situations. Double bevels have higher maximum pressure pulses and the filter cake growth rate 'lifts' the metering blade such that the final coating is left thicker. The model can be used to optimise blade 
geometry to reduce operational anomalies such as streaking and scratching, which are a function of coating colour stagnation.

\section{References}

[1] Linnnonmaa, J. \& Trefz, M., Pigment coating techniques. In: E. Lehtinen, ed. Pigment coating and surface sizing of paper. Helsinki, Finland: Fapet, 414-486, 2000.

[2] Klass, C.P., Update on Coated Papers, Coating Processes and Materials, China Paper 2005, Beijing China, September 19, 2005.

[3] Lehtinen, E., Introduction to pigment coating of paper. In: E. Lehtinen, ed. Pigment coating and surface sizing of paper. Helsinki, Finland: Fapet, 1327, 2000.

[4] Weigl, J. \& Grossmann, H., Investigation into the runability of coating colours at the blade at high production speeds. In: 1996 coating conference, 19-22 May 1996 Nashville, TN. Atlanta, USA: Tappi Press, 311-321, 1996.

[5] Smith, R.D., Roll and web defect terminology, Tappi, 1995.

[6] Alam, P. \& Toivakka, M., Micro-buckling of paper during blade metering, Computers and Chemical Engineering, 32, 600-607, 2008.

[7] Alam, P. \& Toivakka, M., The interfacial slippage of paper during blade metering, Proceedings of the European Coating Symposium 2005, Bradford, UK, 2005.

[8] Daiß, A., Wagner, H-G. \& Wirth, T., Flow structures within the distribution chamber of a high speed short dwell coater - Numerical investigation and comparison with experimental data, TAPPI Coating Conference, 1998.

[9] Tönük, E. \& Ünlüsoy, Y. S., Prediction of automobile tire cornering force characteristics by finite element modeling and analysis, Computers and Structures, 79, 1219-1232, 2001.

[10] Pajari, H., Mansikka-Aho, J., Ketoja, J. \& Bousfield, D.W., Blade coating with free jet applicator: modeling and experiments, Proceedings of the 8th Advanced Fundamentals Symposium, TAPPI Press, 2003.

[11] Mann, R.W., Baum, G.A. \& Habeger, C.C., Determination of all nine orthotropic elastic constants for machine-made paper, Tappi, 63, 163-166, 1980.

[12] Alam, P. \& Toivakka, M., Micro-buckling of paper during blade metering, Computers and Chemical Engineering, 62, 12, 3142-3158, 2007.

[13] Bousfield, D.W., Rigdahl, M. \& Wikström, M., Roll deformation during blade coating. Tappi, 81 (5), 207-212, 1998.

[14] Saita, F.A. \& Scriven, L.E., Coating flow analysis and the physics of flexible blade coating. Coating conference proceedings, Atlanta, USA: Tappi Press, 1985.

[15] Iliopoulos, I. \& Scriven, L. E., A blade-coating study using a finite-element simulation, Physics of Fluids, 17, 1-17, 2005. 